\title{
Article
}

\section{Dual Numbers and Operational Umbral Methods}

\author{
Nicolas Behr ${ }^{1, *(\mathbb{D})}$, Giuseppe Dattoli ${ }^{2}$, Ambra Lattanzi ${ }^{2,3}$ (1) and Silvia Licciardi ${ }^{2}$ (1) \\ 1 Institut de Recherche en Informatique Fondamentale (IRIF), Université de Paris, Bâtiment Sophie Germain, \\ Case Courier 7014, 8 Place Aurélie Nemours, CEDEX 13, 75205 Paris, France \\ 2 ENEA-Frascati Research Center, Via Enrico Fermi 45, 00044 Rome, Italy \\ 3 H. Niewodniczański Institute of Nuclear Physics, Polish Academy of Science, ul. Radzikowskiego 152, \\ 31-342 Kraków, Poland \\ * Correspondence: nicolas.behr@irif.fr
}

Received: 22 May 2019; Accepted: 26 June 2019; Published: 2 July 2019

check for updates

\begin{abstract}
Dual numbers and their higher-order version are important tools for numerical computations, and in particular for finite difference calculus. Based on the relevant algebraic rules and matrix realizations of dual numbers, we present a novel point of view, embedding dual numbers within a formalism reminiscent of operational umbral calculus.
\end{abstract}

Keywords: dual numbers; operational methods; umbral image techniques

\section{Introduction}

Dual numbers (DNs), introduced during the second half of the 19th century [1-5], can be viewed as abstract entities, similar to ordinary complex numbers, and are defined as

$$
z=x+\epsilon y, \quad(x, y) \in \mathbb{R}
$$

where the corresponding "imaginary" unit or dual number unit $(D N U) \epsilon$ is a nilpotent number,

$$
\epsilon^{2}=0 \quad \text { and } \quad \epsilon \neq 0
$$

Dual numbers were originally introduced within the context of geometrical studies, and later exploited to deal with problems in pure and applied mechanics [6,7]. For instance, it has been demonstrated in [8-10] how to formulate the equations of rigid body motion in terms of just three "dual" equations instead of their six "real" counterparts (thereby realizing an equivalence between spherical and spatial kinematics). This approach has been extended in [11-13] to a treatment of rigid body motion in terms of a certain variant of "hyper-dual" numbers, implemented in contrast to our approach via sets of "ordinary" dual numbers together with certain algebraic relations that are motivated from the specific requirements within the relevant field of robotics and of mechanics. More recently, as further discussed in the present paper, the importance of a different kind of higher-order dual numbers has been recognized in numerical analysis to reduce round-off errors [14]. We believe that the use of dual numbers in the applied sciences is not as widespread as it could be, and that many new fields of research would benefit from their relevant introduction. An important domain in which they may bring significant novelties is that of the perturbative techniques in classical and quantum mechanics.

The main contribution of this paper consists in fixing the underlying algebraic rules of the dual numbers in the wider context of umbral and operational calculus. The paper is organized as follows: Section 2 delivers a basic mathematical introduction to dual numbers. Section 3 is devoted to the description of the computational procedure based upon dual numbers and umbral calculus. In Section 4, we provide insight into how this powerful method can be applied to deal with 
problems arising in different contexts. For illustration, we consider the Schrödinger and the heat equation, cornerstones in their respective fields of physics. Section 5 provides a conclusion with further considerations for future works.

\section{Higher-Order Dual Numbers}

The DN algebraic rules [15,16], summarized below, are a straightforward consequence of the previous identity in Equation (2) (with $z=x+\epsilon y$ and $w=u+\epsilon v$ ):

\section{Component-wise algebraic addition}

$$
z+w=x+u+\epsilon(y+v)
$$

\section{Product}

$z \cdot w=x u+\epsilon(x v+y u)$

Inverse

$$
z^{-1}=\frac{1}{x}\left(1-\epsilon \frac{y}{x}\right) \quad(x \neq 0)
$$

\section{Power}

$$
z^{n}=x^{n}\left(1+n \epsilon \frac{y}{x}\right) \quad\left(n \in \mathbb{Z}_{\geq 0}, x \neq 0\right)
$$

While the addition operation is entirely analogous to the component-wise addition operation on two-dimensional vectors, the last three operations (product, inverse and power) characterize the distinguishing special algebraic properties of dual numbers (DNs). The multiplication is commutative, associative and distributive, thus DNs form a two-dimensional associative and commutative algebra over the real numbers.

We now extend this traditional dual number formalism as motivated by the following type of problem. Consider the Taylor expansion up to some order $k$ (denoted $\approx_{k}$ ) of an at least $k$-fold continuously differentiable function $f$ around a point $x$,

$$
f(x+y) \approx_{k} \sum_{m=0}^{k} \frac{y^{m}}{m !} f^{(m)}(x) .
$$

Following the automatic differentiation paradigm [17-19], since in practice the function $f$ will be implemented in some algorithmic from, it may be advantageous to formulate truncations such as Equation (4) in terms of generalized (or higher-order) dual numbers. To this end, let us introduce the families of square matrices ${ }_{k} \hat{\epsilon}_{ \pm}, \hat{k}^{\hat{1}}$ and ${ }_{k} \hat{0}$ with entries (for $i, j=1, \ldots, k$ )

$$
\left({ }_{k} \hat{\epsilon}_{ \pm}\right)_{i, j}:=\delta_{j, i \pm 1}, \quad\left({ }_{k} \hat{1}\right)_{i, j}:=\delta_{i, j}, \quad\left({ }_{k} \hat{0}\right)_{i, j}:=0,
$$

where $\delta_{i, j}$ denotes the Kronecker symbol. It is straightforward to verify that for all $k \geq 2$ and $\ell \geq 0$

$$
\left({ }_{k} \hat{\epsilon}_{ \pm}^{\ell}\right)_{i, j}=\delta_{j, i \pm \ell} \Rightarrow\left({ }_{k} \hat{\epsilon}_{ \pm}\right)^{k}=0
$$

Then, under the assumptions in Equation (4), endowing the function $f(x)$ suitably with a component-wise action on square matrices, we find (for $k \geq 2$ )

$$
f\left(x_{k} \hat{1}+y_{k} \hat{\epsilon}_{ \pm}\right)=\sum_{m=0}^{k-1} \frac{1}{m !} y^{m} f^{(m)}(x)_{k} \hat{\epsilon}_{ \pm}^{m} .
$$


For example, setting $k=2$, reproduces the well-known dual number identity [19]

$$
f\left(x_{2} \hat{1}+y_{2} \hat{\epsilon}_{+}\right)=f(x)_{2} \hat{1}+y f^{\prime}(x)_{2} \hat{\epsilon}_{+}=\left(\begin{array}{cc}
f(x) & y f^{\prime}(x) \\
0 & f(x)
\end{array}\right) .
$$

For illustration, setting $k=3$, we obtain

$$
f\left(x_{3} \hat{1}+y_{3} \hat{\epsilon}_{+}\right)=f(x)_{3} \hat{1}+y f^{\prime}(x)_{3} \hat{\epsilon}_{+}+\frac{1}{2} y^{2} f^{\prime \prime}(x)_{3} \hat{\epsilon}_{+}^{2}=\left(\begin{array}{ccc}
f(x) & y f^{\prime}(x) & \frac{1}{2} y^{2} f^{\prime \prime}(x) \\
0 & f(x) & y f^{\prime}(x) \\
0 & 0 & f(x)
\end{array}\right) .
$$

It may be verified that, e.g., for the choice "+" in Equation (5), the first row of the resulting matrices in Equation (7) contain the terms of the Taylor expansion up to order $k-1$. More explicitly, introducing the auxiliary notations for the row vector $\left\langle{ }_{k} e_{1}\right|$ and the column vector $\left|{ }_{k} \mathbf{1}\right\rangle$ of length $k \geq 2$,

$$
\left\langle{ }_{k} e_{1}|:=(1,0, \ldots, 0), \quad|{ }_{k} \mathbf{1}\right\rangle:=\left(\begin{array}{c}
1 \\
\vdots \\
1
\end{array}\right),
$$

allows us to define the order $k$ evaluation operation acting on some function $F\left({ }_{k} \hat{\epsilon}_{+}\right)$(which is itself assumed to act entry-wise) depending on a generalized dual number ${ }_{k} \hat{\epsilon}_{+}$as

$$
\left\langle F\left({ }_{k} \hat{\epsilon}_{+}\right)\right\rangle_{k}:=\left\langle{ }_{k} e_{1}\left|F\left({ }_{k} \hat{\epsilon}_{+}\right)\right|{ }_{k} \mathbf{1}\right\rangle .
$$

We thus find that

$$
\left\langle f\left(x_{k} \hat{1}+y_{k} \hat{\epsilon}_{+}\right)\right\rangle_{k}=\sum_{m=0}^{k-1} \frac{y^{m}}{m !} f^{(m)}(x) .
$$

Recently, expansions such as Equation (7) have received considerable interest in the field of numerical analysis [20]. Referring to Fike [19] for an overview, various alternative types of "numbers" have been studied for the purpose of finding optimized numerical schemes for computing $k$ th-order derivatives of functions. For example, it has been demonstrated that the use of so-called hyper-dual numbers results in first- and second-derivative calculations that are exact, regardless of the step size [14].

For later convenience, motivated by the identity (for $k \geq 2$ )

$$
\exp \left({ }_{k} \hat{\epsilon}_{+} x\right)=\sum_{r=0}^{k-1} \frac{x^{r}}{r !} \hat{\epsilon}_{+}^{r},
$$

we may introduce the so-called truncated exponential polynomials [21] $e_{n}(x)$ defined through the series

$$
e_{n}(x):=\sum_{r=0}^{n} \frac{x^{r}}{r !}
$$

which may be expressed in terms of generalized dual numbers as

$$
\left.\left(\begin{array}{c}
e_{n}(x) \\
e_{n-1}(x) \\
\vdots \\
e_{1}(x) \\
1
\end{array}\right):=\left.\exp \left({ }_{n+1} \hat{\epsilon}_{+} x\right)\right|_{n+1} \mathbf{1}\right\rangle
$$


One may thus easily verify (via Equations (13) and (14)) the property

$$
e_{n}^{\prime}(x)=e_{n-1}(x) .
$$

Having provided a matrix-based extension of ordinary to $k$ th-order dual numbers of arbitrary order $k \geq 2$, we now proceed to develop a computational procedure embedding dual numbers with other techniques inspired by the operational umbral formalism.

\section{Umbral-Type Methods and Dual Numbers}

Starting from this section, we employ the notational simplification of writing $\epsilon$ for the dual number unit (DNU) ${ }_{k} \hat{\epsilon}_{ \pm}$of generalized dual numbers (cf. Equation (5)), making the order $k \geq 2$ of the DN explicit only via the analog of the notation in Equation (11), and masking the matrix nature of ${ }_{k} \hat{\epsilon}_{ \pm}$. Thus, for some function $F \equiv F(\epsilon)$, we write

$$
F \rightsquigarrow_{k} G \quad: \Leftrightarrow \quad G=\left.\left(\left.F\right|_{\epsilon^{k+1} \rightarrow 0}\right)\right|_{\epsilon \rightarrow 1}
$$

for the truncation of $F$ via setting $\epsilon^{k+1}=0$ and afterwards $\epsilon=1$. It is straightforward to verify that this formal definition may be implemented in terms of the matrix representations introduced in Section 2 via use of Equation (11) as

$$
G=\left\langle F\left({ }_{k+1} \hat{\epsilon}_{+}\right)\right\rangle_{k+1} .
$$

Consider then the dual complex parameter

$$
\hat{z} \equiv \hat{z}(a, b):=a+\epsilon b .
$$

Following the principles of umbral calculus, we treat the dual complex parameter $\hat{z}$ as an ordinary algebraic quantity in calculations of integrals, derivatives and other operations, delaying the evaluation of $\hat{z}$ via performing the operation $\rightsquigarrow_{k}$ to the very end of the computations. Note that albeit the term umbral calculus has been introduced in the seminal papers by Roman and Rota [22], in the following we make reference to the formalism developed in [23] which enriches the original formalism with the wealth of techniques derived from the operational calculus [22,23]. We now illustrate the computational benefits of this approach via a number of examples.

\subsection{Dual Shifted Gaussians}

We first consider a Gaussian-type function explicitly containing in its argument the dual complex parameter in Equation (19), whence the dual-shifted Gaussian function

$$
f(x)=e^{-\alpha x^{2}+\hat{z}(a, b) x} .
$$

Assuming for instance third-order dual numbers (i.e., $\epsilon^{3}=0$ ), we may write the above function in more conventional terms as

$$
f(x) \rightsquigarrow 2 e^{-\alpha x^{2}+a x}\left[1+b x+\frac{1}{2}(b x)^{2}\right],
$$

which is easily recognized as the product of a shifted Gaussian with a second-degree polynomial.

In full analogy to the umbral operational methods of Licciardi [23], it is then straightforward to calculate the following integral of the function $f$ of Equation (20) via the standard Gaussian integral formula

$$
\int_{-\infty}^{+\infty} f(x) d x=\sqrt{\frac{\pi}{\alpha}} e^{\frac{z(a, b)^{2}}{4 \alpha}}=\sqrt{\frac{\pi}{\alpha}} e^{\frac{a^{2}}{4 \alpha}+\frac{a b}{2 \alpha} \epsilon+\frac{b^{2}}{4 \alpha} \epsilon^{2}} .
$$


The term on the right has in fact a definite meaning, since the use of the generating function of the two-variable Hermite polynomials [24]

$$
\begin{aligned}
\sum_{n=0}^{\infty} \frac{t^{n}}{n !} H_{n}(x, y) & =e^{x t+y t^{2}} \\
H_{n}(x, y) & =e^{y \partial_{x}^{2}} x^{n}=n ! \sum_{r=0}^{\left\lfloor\frac{n}{2}\right\rfloor} \frac{x^{n-2 r} y^{r}}{(n-2 r) ! r !}
\end{aligned}
$$

permits to cast the right-hand side of Equation (22) into the form

$$
\sqrt{\frac{\pi}{\alpha}} e^{\frac{a^{2}}{4 \alpha}+\frac{a b}{2 \alpha} \epsilon+\frac{b^{2}}{4 \alpha} \epsilon^{2}}=\sqrt{\frac{\pi}{\alpha}} e^{\frac{a^{2}}{4 \alpha}} \sum_{m \geq 0} \frac{\epsilon^{m}}{m !} H_{m}\left(\frac{a b}{2 \alpha}, \frac{b^{2}}{4 \alpha}\right) \rightsquigarrow_{k} \sqrt{\frac{\pi}{\alpha}} e^{\frac{a^{2}}{4 \alpha}} H e_{k}\left(\frac{a b}{2 \alpha}, \frac{b^{2}}{4 \alpha}\right) .
$$

Here, ${ }_{H} e_{k}(x, y)$ denotes the Hermite-based truncated exponential polynomial [25-27] defined as

$$
{ }_{H} e_{k}(x, y):=\sum_{r=0}^{k} \frac{1}{r !} H_{r}(x, y)
$$

\subsection{Another Form of Dual Gaussian}

Let us consider as a further example

$$
g(x):=e^{-\hat{z}(a, b) x^{2}}
$$

and the following infinite integral (for $\operatorname{Re}(a)>0$ )

$$
\int_{-\infty}^{+\infty} g(x) d x=\sqrt{\frac{\pi}{\hat{z}}}=\sqrt{\frac{\pi}{a+\epsilon b}} \rightsquigarrow k \sqrt{\frac{\pi}{a}} \sum_{r=0}^{k}\left(\begin{array}{c}
-\frac{1}{2} \\
r
\end{array}\right)\left(\frac{b}{a}\right)^{r} .
$$

Here, by invoking the operation $\rightsquigarrow_{k}$, we obtain a finite series, thus obviating the need to impose any condition on the relevant convergence range.

\subsection{Examples From Symbolic Calculus}

The calculus of higher-order dual numbers may be further refined via combining it with the wealth of techniques available from the theory of special functions and symbolic calculus as put forward in [23,28-32]. Consider for illustration the following identity, known from the theory of two-variable Hermite polynomials [33],

$$
\partial_{x}^{n} e^{\alpha x^{2}}=H_{n}(2 \alpha x, \alpha) e^{\alpha x^{2}}
$$

which allows to simplify the task of calculating successive derivatives of the dual Gaussian introduced in Equation (26), such as in the computation

$$
\partial_{x}^{n} e^{-\hat{z} x^{2}}=H_{n}(-2 \hat{z} x,-\hat{z}) e^{-\hat{z} x^{2}}=n ! \sum_{r=0}^{\left\lfloor\frac{n}{2}\right\rfloor} \sum_{s \geq 0} \frac{(-1)^{n-r+s} 2^{n-2 r} x^{n-2(r-s)}}{(n-2 r) ! r ! s !} \hat{z}^{n-r+s} .
$$

Here, the first step follows from (28) and the second by invoking (23b).

Another interesting type of calculus concerns infinite integrals involving rational functions such as

$$
\Phi(x ; a, b):=\frac{1}{1+\hat{z} x^{2}} \rightsquigarrow_{k} \frac{1}{1+a x^{2}} \sum_{r=0}^{k}\left(-\frac{b x^{2}}{1+a x^{2}}\right)^{r} .
$$


For example, the infinite integral

$$
\int_{-\infty}^{+\infty} \frac{1}{1+\hat{z} x^{2}} d x=\frac{\pi}{\sqrt{\hat{z}}}
$$

may be easily transformed into truncated form in full analogy to the calculation summarized in Equation (27).

\subsection{Umbral Image Type Techniques}

Referring to Behr et al. [34] for the precise technical details (compare also [33]), suffice it here to provide the following definition for the action of the formal integration operator $\hat{\mathbb{I}}$ on the formal variable $v($ for $\alpha \in \mathbb{C})$ :

$$
\hat{\mathbb{I}}\left(v^{\alpha}\right):=\frac{1}{\Gamma(\alpha)} .
$$

Then, an interesting variant of the example presented in Equation (29) may be obtained as

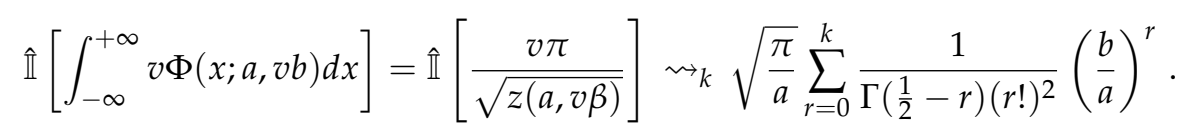

In summary, the combination of the concept of higher order dual numbers with techniques from symbolic and umbral-image type calculus appears to offer a large potential in view of novel tools of computation. To corroborate this claim, we now present some first high-level results in this direction.

\section{Dual Numbers and Solution of Heat- and Schrödinger-Type Equations}

Before entering the main topic of this section, let us recall a few useful "operational rules", starting with the Glaisher identity $[27,35]$

$$
e^{\tau \frac{d^{2}}{d x^{2}}} e^{-\alpha x^{2}}=\frac{1}{\sqrt{1+4 \tau \alpha}} e^{-\frac{\alpha x^{2}}{1+4 \tau \alpha}}
$$

which can also be understood as the solution of the heat equation with a Gaussian as initial function. It proves particularly useful in the following to note that, according to the definition of the Hermite polynomials $H_{n}(x, y)$ as given in Equation (23b), an alternative interpretation of Equation (33) is provided in terms of the double-lacunary exponential generating function $\mathcal{H}_{2,0}(\lambda ; x, y)$ of the polynomials $H_{n}(x, y)$, where we employ notations as in [36]

$$
e^{\tau \frac{d^{2}}{d x^{2}}} e^{-\alpha x^{2}}=\sum_{n \geq 0} \frac{(-\alpha)^{n}}{n !} H_{2 n}(x, \tau)=\mathcal{H}_{2,0}(-\alpha ; x, \tau) .
$$

By specializing Equation (33) to $\alpha=\hat{z}$ (with $\hat{z}=a+\epsilon b$ the dual complex parameter in Equation (19)), we obtain the operational identity

$$
e^{\tau \frac{d^{2}}{d x^{2}}} e^{-\hat{z} x^{2}}=\frac{1}{\sqrt{1+4 \tau \hat{z}}} e^{-\frac{\hat{z} x^{2}}{1+4 \tau \hat{z}}}
$$

Via the simple factorizations

$$
\begin{aligned}
1+4 \hat{z} \tau & =\gamma(a, \tau) \gamma\left(\frac{b \epsilon}{\gamma(a, \tau)}, \tau\right) \\
\frac{\hat{z}}{1+4 \hat{z} \tau} & =\frac{a}{\gamma(a, \tau)}+\frac{b \epsilon}{[\gamma(a, \tau)]^{2} \gamma\left(\frac{b \epsilon}{\gamma(a, \tau)}, \tau\right)} \\
\gamma(c, \tau) & =1+4 c \tau
\end{aligned}
$$


we may transform the identity in Equation (35) as

$$
e^{\tau \frac{d^{2}}{d x^{2}}} e^{-\hat{z} x^{2}}=\mathcal{H}_{2,0}\left(-\frac{b \epsilon}{[\gamma(a, \tau)]^{2}} ; x, \tau \gamma(a, \tau)\right) \mathcal{H}_{2,0}(-a ; x, \tau)
$$

By re-inserting the definition of the first double-lacunary exponential generating function, using the Glaisher-identity in Equation (33) for the second one and finally truncating to order $k$, we eventually arrive at the compact result

$$
e^{\tau \frac{d^{2}}{d x^{2}}} e^{-\hat{z} x^{2}} \rightsquigarrow k \frac{e^{-\frac{\alpha x^{2}}{\gamma(a, \tau)}}}{\sqrt{\gamma(a, \tau)}} \sum_{n=0}^{k} \frac{1}{n !}\left(\frac{b}{[\gamma(a, \tau)]^{2}}\right)^{n} H_{2 n}(x, \tau \gamma(a, \tau)), \quad \gamma(a, \tau)=1+4 a \tau .
$$

For example, by evaluating the above expression for second order dual numbers, one finds

$$
e^{\tau \frac{d^{2}}{d x^{2}}} e^{-\hat{z} x^{2}} \rightsquigarrow 2 \frac{e^{-\frac{a x^{2}}{\gamma(a, \tau)}}}{\sqrt{\gamma(a, \tau)}}\left(1-\frac{b}{\gamma(a, \tau)^{2}} H_{2}(x, \tau \gamma(a, \tau))+\frac{b^{2}}{2 \gamma(a, \tau)^{4}} H_{4}(x, \tau \gamma(a, \tau))\right) .
$$

The above result may be interpreted as the solution of the heat-type equation

$$
\partial_{\tau} F(x, \tau)=\partial_{x}^{2} F(x, \tau), \quad F(x, 0)=e^{-\hat{z} x^{2}} .
$$

An analogous problem has been addressed in [33] within the framework of a different method. The techniques we have envisaged may be further exploited to treat the paraxial propagation of the so-called flattened distributions, introduced in [37] to study the laser field evolution in optical cavities employing super-Gaussian mirrors [38]. These cavities shape beams whose transverse distribution is not reproduced by a simple Gaussian, but by a function exhibiting a quasi-constant flat-top, expressible through a function of the type

$$
E(x ; p):=e^{-|x|^{p}}, \quad p \in \mathbb{Z}_{>0} .
$$

The paraxial propagation of these beams has less obvious properties than, e.g., Laguerre or Hermite Gauss modes [38]. To overcome this drawback, Gori introduced the so-called flattened beams [37], which permit a fairly natural expansion in terms of Gauss Laguerre/Hermite modes, thus providing a straightforward solution to the corresponding paraxial wave equation.

Invoking our formalism as developed thus far, we may approximate the aforementioned Gori beams in the form

$$
E(x ; p) \approx Y(x ; \alpha \mid m):=e^{-\alpha x^{2}} e_{m}\left(x^{2}\right) .
$$

Here, $e_{m}(x)$ denotes the truncated exponential polynomials introduced in Equation (14), and both parameters $\alpha$ and $m$ depend on $p$ (see [33] for further details). Recalling from Equation (19) the definition $\hat{z}(a, b):=a+b \epsilon$ of the dual complex parameter, the r.h.s. of Equation (42) may be equivalently expressed as

$$
e^{-\hat{z}(\alpha,-1) x^{2}} \rightsquigarrow m Y(x ; \alpha \mid m),
$$

whence as an instance of a dual Gaussian as described in Section 3.2. The problem of the relevant propagation can accordingly be reduced to that of an ordinary Gaussian mode, namely to the solution of the Schrödinger type equation

$$
i \partial_{\tau} \Psi(x, \tau)=-\partial_{x}^{2} \Psi(x, \tau), \quad \Psi(x, 0)=Y(x ; \alpha \mid m) .
$$


Consequently, by invoking the operational identity in Equation (35), the paraxial evolution of a flattened beam may be expressed in the form

$$
\Psi(x, \tau)=e^{i \tau \partial_{x}^{2}} e^{-\hat{z}(\alpha,-1) x^{2}}=\frac{1}{\sqrt{1+4 i \tau \hat{z}(\alpha,-1)}} e^{-\frac{\hat{z}(\alpha,-1) x^{2}}{1+4 i \tau z(\alpha,-1)}},
$$

which reproduces indeed the known solution of our problem (compare [33]).

In a forthcoming paper, we will discuss this specific application in further detail by applying the method to the problem of designing super-Gaussian optical systems.

\section{Weyl Formula and Modified Hermite Polynomials}

The wide flexibility of the method we propose is corroborated by the following further example, relevant to the use of operational ordering tools. Let us consider an evolution equation of the form

$$
\partial_{\tau} F(x, \tau)=\left[\gamma \partial_{x}-\hat{z} x\right] F(x, \tau), \quad F(x, 0)=f(x) .
$$

The relevant procedure for combining differential calculus with the umbral formalism is described in [39]. Following this approach, the solution of Equation (46) can be expressed as

$$
F(x, \tau)=e^{\tau\left(\gamma \partial_{x}-2 x\right)} f(x) .
$$

To evaluate the solution of Equation (47) explicitly, we need to suitably "factorize" the exponential operator. This so-called disentanglement operation may be implemented via the Weyl formula [40]

$$
e^{\hat{X}+\hat{Y}}=e^{-\frac{1}{2}[\hat{X}, \hat{Y}]} e^{\hat{X}} e^{\hat{Y}}
$$

which is applicable whenever the identities $[\hat{X},[\hat{X}, \hat{Y}]]=[[\hat{X}, \hat{Y}], \hat{Y}]]=0$ hold. Applying the Weyl formula in Equation (48) to our solution in Equation (47), if we let $\hat{X}=-\tau \hat{z} x$ and $\hat{Y}=\tau \gamma \partial_{x}$ (resulting in $[\hat{X}, \hat{Y}]=\tau^{2} \gamma \hat{z}$, and with higher-order commutators vanishing), we obtain

$$
F(x, \tau)=e^{-\frac{1}{2} \tau^{2} \gamma z} e^{-z x \tau} f(x+\gamma \tau) .
$$

Thus, the solution at any desired truncation order $k$ may be obtained by invoking the dual number evaluation operation $\rightsquigarrow_{k}$ of Equation (17).

As already mentioned above, the Weyl formula applies in the example presented because the algebraic structure of the argument of the exponential in Equation (47) satisfies a special property: the commutators of the associated generators reduce to a constant after the first commutation bracket. A more interesting extension is given by the case in which the generators are embedded into a solvable Lie algebra. In this case, the combined use of the dual number formalism and of the Wei-Norman ordering method [41] leads to new and interesting results. They deserve a separate treatment that will be reported in a forthcoming paper.

As a final example, we define modified Hermite polynomials $H_{n}(x, \hat{z})$, whence ordinary two-variable Hermite polynomials $H_{n}(x, y)$ as introduced in Equation (23b) evaluated at $y=\hat{z}$, with $\hat{z} \equiv \hat{z}(a, b)$ the dual complex parameter of Equation (19),

$$
H_{n}(x, \hat{z})=e^{\imath \partial_{x}^{2}} x^{n} .
$$

It is straightforward to verify that these modified polynomials inherit all the relevant properties from the polynomials $H_{n}(x, y)$, such as the recurrences

$$
\begin{aligned}
& \partial_{x} H_{n}(x, \hat{z})=n H_{n-1}(x, \hat{z}), \\
& H_{n+1}(x, \hat{z})=x H_{n}(x, \hat{z})+2 \hat{z} \partial_{x} H_{n}(x, \hat{z}),
\end{aligned}
$$


and we find that they satisfy the second order differential equation

$$
2 z \partial_{x}^{2} H_{n}(x, \hat{z})+x \partial_{x} H_{n}(x, \hat{z})=n H_{n}(x, \hat{z}) .
$$

The explicit form of these truncated polynomials is easily obtained. For example, by using third order dual numbers, which implies

$$
e^{\hat{z} \partial_{x}^{2}} \rightsquigarrow_{2} e^{a \partial_{x}^{2}}\left(1+b \partial_{x}^{2}+\frac{1}{2} b^{2} \partial_{x}^{4}\right),
$$

we find the explicit formula

$$
H_{n}(x, \hat{z}) \rightsquigarrow 2 H_{n}(x, a)+b \partial_{a} H_{n}(x, a)+\frac{1}{2} b^{2} \partial_{a}^{2} H_{n}(x, a),
$$

where we have invoked the well-known identity

$$
\partial_{x}^{2} H_{n}(x, y)=\partial_{y} H_{n}(x, y) .
$$

\section{Final Comments}

The method we have outlined in this paper offers many computational advantages to treat problems where truncated expansions (not necessarily of Taylor type) of functions are involved. At its core, the umbral formalism and the notion of higher order dual numbers allow delaying the explicit expansions to later stages in a given calculation, thus opening the possibility to exploit numerous efficient computation strategies from the theory of operational calculus and special functions.

The technique we have introduced in this paper is amenable for new applications in various different fields. We have presented herein the solution of parabolic equations in transport problems, and within such a context a fairly important example has been provided by treating the propagation of flattened beams [33,37] in optics. For brevity, we have just outlined the procedure in terms of a one-dimensional computation. The relevant extension to the three-dimensional case does not require any particular conceptual effort, but only a consistent numerical implementation. In a forthcoming investigation, we will further extend the method and study its potential for treating perturbative problems in classical and quantum mechanics.

Author Contributions: Conceptualization, G.D.; methodology, N.B. and G.D.; validation, N.B., G.D. and S.L.; formal analysis, N.B., G.D., A.L., and S.L.; writing-original draft preparation, N.B., G.D., and A.L.; and writing-review and editing, N.B., A.L., and S.L.

Funding: The work of N.B. is supported by funding from the European Union's Horizon 2020 research and innovation programme under the Marie Skłodowska-Curie grant agreement No. 753750. A.L. was supported by the NCN research project OPUS 12 No. UMO-2016/23/B/ST3/01714 and by the NAWA project: Program im. Iwanowskiej PPN/IWA/2018/1/00098. S.L. was supported by an Enea-Research Center Individual Fellowship.

Acknowledgments: N.B. would like to thank the LPTMC (Paris 06) and ENEA Frascati for warm hospitality.

Conflicts of Interest: The authors declare no conflict of interest. The founding sponsors had no role in the design of the study; in the collection, analyses, or interpretation of data; in the writing of the manuscript, and in the decision to publish the results.

\section{References}

1. Clifford, W.K. Preliminary Sketch of Biquaternions. Proc. Lond. Math. Soc. 1871, s1-s4, 381-395. [CrossRef]

2. Grünwald, J. Über duale Zahlen und ihre Anwendung in der Geometrie. Monatshefte für Mathematik Physik 1906, 17, 81-136. [CrossRef]

3. Segre, C. Le Geometrie Projettive nei Campi di Numeri Duali; Vincenzo Bona: Torino, Italy, 1912.

4. Yaglom, I.M. A Simple Non-Euclidean Geometry and Its Physical Basis: An Elementary Account of Galilean Geometry and the Galilean Principle of Relativity; Springer: Berlin, Germany, 2012. 
5. Klawitter, D. Clifford Algebras: Geometric Modelling and Chain Geometries with Application in Kinematics; Springer: Berlin, Germany, 2014.

6. Kotelnikov, A.P. Screw Calculus and Some Applications to Geometry and Mechanics. Ann. Imp. Univ. Kazan 1895, 24. (In Russian)

7. Study, E. Die Geometrie der Dynamen. Jahresbericht der Deutschen Mathematiker-Vereinigung 1900, 8, $204-216$.

8. Rooney, J. On the principle of transference. In Proceedings of the Fourth World Congress on the Theory of Machines and Mechanisms; Institution of Mechanical Engineers: London, UK, 1975; pp. 1088-1092.

9. Hsia, L.; Yang, A. On the principle of transference in three-dimensional kinematics. J. Mech. Des. 1981, 103, 652-656. [CrossRef]

10. Martínez, J.M.R.; Duffy, J. The principle of transference: History, statement and proof. Mech. Mach. Theory 1993, 28, 165-177. [CrossRef]

11. Cohen, A.; Shoham, M. Application of Hyper-Dual Numbers to Multibody Kinematics. J. Mech. Rob. 2015, 8, 011015. [CrossRef]

12. Cohen, A.; Shoham, M. Application of hyper-dual numbers to rigid bodies equations of motion. Mech. Mach. Theory 2017, 111, 76-84. [CrossRef]

13. Cohen, A.; Shoham, M. Principle of transference-An extension to hyper-dual numbers. Mech. Mach. Theory 2018, 125, 101-110. [CrossRef]

14. Fike, J.; Alonso, J. The Development of Hyper-Dual Numbers for Exact Second-Derivative Calculations. In Proceedings of the 49th AIAA Aerospace Sciences Meeting including the New Horizons Forum and Aerospace Exposition; American Institute of Aeronautics and Astronautics: Reston, VA, USA, 2011. [CrossRef]

15. Harkin, A.A.; Harkin, J.B. Geometry of Generalized Complex Numbers. Math. Mag. 2004, 77, 118-129. [CrossRef]

16. Özdemir, M. Introduction to Hybrid Numbers. Adv. Appl. Clifford Algebr. 2018, 28. [CrossRef]

17. Rall, L.B. (Ed.) Automatic Differentiation: Techniques and Applications; Springer: Berlin/Heidelberg, Germany, 1981. [CrossRef]

18. Rall, L.B.; Corliss, G.F. An introduction to automatic differentiation. In Computational Differentiation: Techniques, Applications, and Tools; SIAM: Philadelphia, PA, USA, 1996, Volume 89.

19. Fike, J.A. Derivative Calculations Using Hyper-Dual Numbers; Technical Report; Sandia National Lab. (SNL-NM): Albuquerque, NM, USA, 2016.

20. Berland, H. (Department of Mathematical Sciences, NTNU, Trondheim, Norway). Personal communication, 2006.

21. Dattoli, G.; Cesarano, C.; Sacchetti, D. A note on truncated polynomials. Appl. Math. Comput. 2003, 134, 595-605. [CrossRef]

22. Roman, S.M.; Rota, G.C. The umbral calculus. Adv. Math. 1978, 27, 95-188. [CrossRef]

23. Licciardi, S. Umbral Calculus, a Different Mathematical Language. arXiv 2018, arXiv:1803.03108.

24. Appel, P.; De Feriet, J.K. Fonctions hypergéométriques et hypersphériques. In Polynômes d'Hermite; Gauthier-Villars: Paris, France, 1926.

25. Dattoli, G. Hermite-Bessel and Laguerre-Bessel functions: A by-product of the monomiality principle. In Proceedings of the Melfi School on Advanced Topics in Mathematics and Physics, Advanced Special Functions and Applications; Aracne Editrice: Melfi, Italy, 2000; pp. 147-164.

26. Dattoli, G. Generalized polynomials, operational identities and their applications. J. Comput. Appl. Math. 2000, 118, 111-123. [CrossRef]

27. Dattoli, G.; Khan, S.; Ricci, P. On Crofton-Glaisher type relations and derivation of generating functions for Hermite polynomials including the multi-index case. Integral Transf. Spec. Funct. 2008, 19, 1-9. [CrossRef]

28. Babusci, D.; Dattoli, G.; Górska, K.; Penson, K. Repeated derivatives of composite functions and generalizations of the Leibniz rule. Appl. Math. Comput. 2014, 241, 193-199. [CrossRef]

29. Babusci, D.; Dattoli, G. On Ramanujan Master Theorem. arXiv 2011, arXiv:1103.3947.

30. Górska, K.; Babusci, D.; Dattoli, G.; Duchamp, G.; Penson, K. The Ramanujan master theorem and its implications for special functions. Appl. Math. Comput. 2012, 218, 11466-11471. [CrossRef]

31. Dattoli, G.; Di Palma, E.; Sabia, E.; Gorska, K.; Horzela, A.; Penson, K. Operational versus umbral methods and the Borel transform. Int. J. Appl. Comput. Math. 2017, 3, 3489-3510. [CrossRef]

32. Babusci, D.; Dattoli, G.; Górska, K.; Penson, K. Lacunary generating functions for the Laguerre polynomials. Séminaire Lotharingien Combinatoire 2017, 76, B76b. 
33. Dattoli, G.; Migliorati, M. The truncated exponential polynomials, the associated Hermite forms and applications. Int. J. Math. Math. Sci. 2006, 2006, 98175-1. [CrossRef]

34. Behr, N.; Dattoli, G.; Duchamp, G.; Licciardi, S.; Penson, K. Operational Methods in the Study of Sobolev-Jacobi Polynomials. Mathematics 2019, 7, 124. [CrossRef]

35. Crofton, M. On operative symbols in the differential calculus. Proc. Lond. Math. Soc. 1880, 1, 122-134. [CrossRef]

36. Behr, N.; Duchamp, G.H.; Penson, K.A. Explicit formulae for all higher order exponential lacunary generating functions of hermite polynomials. arXiv 2018, arXiv:1806.08417.

37. Gori, F. Flattened gaussian beams. Opt. Commun. 1994, 107, 335-341. [CrossRef]

38. Siegman, A.E. Lasers university science books. Mill Valley CA 1986, 37, 208.

39. Babusci, D.; Dattoli, G. Umbral methods and operator ordering. arXiv 2011, arXiv:1112.1570.

40. Dattoli, G.; Ottaviani, P.L.; Torre, A.; Vázquez, L. Evolution operator equations: Integration with algebraic and finite difference methods. Applications to physical problems in classical and quantum mechanics and quantum field theory. La Rivista del Nuovo Cimento 1997, 20, 3-133. [CrossRef]

41. Wei, J.; Norman, E. Lie algebraic solution of linear differential equations. J. Math. Phys. 1963, 4, 575-581. [CrossRef]

(C) 2019 by the authors. Licensee MDPI, Basel, Switzerland. This article is an open access article distributed under the terms and conditions of the Creative Commons Attribution (CC BY) license (http://creativecommons.org/licenses/by/4.0/). 